\title{
Supplier Selection Problem Based on Interval Intuitionistic Fuzzy Multiattribute Group Decision Making
}

\author{
Danyi Song, Jiao Wang \\ Beijing Wuzi University, Beijing, China \\ Email: 18801233755@163.com
}

How to cite this paper: Song, D.Y. and Wang, J. (2019) Supplier Selection Problem Based on Interval Intuitionistic Fuzzy Multiattribute Group Decision Making. Open Journal of Business and Management, 7 , 1494-1503.

https://doi.org/10.4236/ojbm.2019.73103

Received: July 9, 2019

Accepted: July 26, 2019

Published: July 29, 2019

Copyright (c) 2019 by author(s) and Scientific Research Publishing Inc. This work is licensed under the Creative Commons Attribution International License (CC BY 4.0).

http://creativecommons.org/licenses/by/4.0/

\begin{abstract}
Aiming at the supplier selection problem where the decision information is an interval intuitionistic fuzzy number and completely does know the attribute and decision maker's weight, this problem is reduced to a multi-attribute group decision problem. A decision method based on information entropy to determine the decision-maker's weight and the deviation maximization method to determine the attribute weight is proposed. Finally, this method is applied to the selection of automotive parts suppliers, and the results are compared with the relevant methods, which fully illustrates the effectiveness of this method.
\end{abstract}

\section{Keywords}

Interval Intuitionistic Fuzzy Numbers, Multi-Attribute Group Decision Making, Fuzzy Entropy, Maximum Dispersion, VIKOR

\section{Introduction}

With the development of economy and society, the competition between enterprises is no longer the unilateral competition between price and quality, but the competition between supply chains. The supplier is at the source of the supply chain and plays a very core role in the whole supply chain. Choosing the right supplier is a good foundation for enterprise development. The evaluation and selection of suppliers is not the individual behavior of purchasers, but actually a complex multi-attribute group decision-making problem. Generally, there are not only quantitative indicators in the index system, such as product price and market share, but also qualitative indicators, such as product research and development capacity, after-sales maintenance level, etc., and the relationship be- 
tween indicators usually has incompatibility and incommensurability. Thus it can be seen that in the process of selecting parts suppliers, enterprises usually have vague and uncertain understanding of suppliers due to the objectivity and complexity of things, and even the effect of evaluation indexes has certain ambiguity, so it is difficult to draw a clear boundary [1], and accurate description becomes impossible. Therefore, the evaluation and selection of suppliers is essentially a fuzzy group decision making problem, which is more suitable to be solved by fuzzy group decision making method, and can effectively improve the scientificity and effectiveness of decision making results. The fuzzy multi-attribute group decision-making problem has been widely studied by many scholars and achieved excellent research results. In practical application, it has also solved many complex and significant problems in fields, such as supplier selection [2]

[3] [4] [5] [6] and risk identification [7] etc.

\section{Knowledge Preparation}

\section{1) Related concepts}

\section{Definition 1 interval intuitionistic fuzzy number [8]}

Set $X$ is A non-empty classic collection, $I_{[0,1]}$ interval $[0,1]$ said on the set of all closed subinterval, for each $x \in X$, said $A=\left\{\left\langle x, U_{A}(x), V_{A}(x)\right\rangle\right\}$ for $A$ range of $X$ on intuitionistic fuzzy sets, including $U_{A}(x)$ and $V_{A}(x)$ are respectively $A$ interval-valued membership functions and interval-valued membership functions, among them, the $U_{A}(x) \in[0,1], V_{A}(x) \in[0,1]$, and meet the conditions $0 \leq \operatorname{Sup}_{A}(x)+\operatorname{Sup}_{A}(x) \leq 1, x \in X$. For simplicity, the upper and lower endpoints of interval value membership $U_{A}(x)$ and interval value non-membership $V_{A}(x)$ are denoated as $U_{A}^{L}(x), U_{A}^{U}(x), V_{A}^{L}(x)$ and $V_{A}^{U}(x)$, respectively. So, for each $x \in X$, interval-valued intuitionistic fuzzy sets $A$ usable form of interval value is expressed as

$A=\left\{\left\langle x,\left[U_{A}^{L}(x), U_{A}^{U}(x)\right],\left[V_{A}^{L}(x), V_{A}^{U}(x)\right]\right\rangle\right\}$. Make

$\pi_{A}(x)=\left[1-U_{A}^{U}(x)-V_{A}^{U}(x), 1-U_{A}^{L}(x)-V_{A}^{L}(x)\right]$ for the element of interval-valued intuitionistic fuzzy sets $A$ 's hesitation.

2) Distance measure of interval intuitionistic fuzzy sets [9]

Definition 2 Let $A$ and $B$ be two interval intuitionistic fuzzy sets, then define the weighted Hamming distance between $A$ and $B$ as:

$$
\begin{aligned}
& d(A, B) \\
& =\frac{1}{4} \sum_{i=1}^{m} \omega_{j}\left[\left|U_{A}^{L}-U_{B}^{L}\right|+\left|U_{A}^{U}-U_{B}^{U}\right|+\left|V_{A}^{L}-V_{B}^{L}\right|+\left|V_{A}^{U}-V_{B}^{U}\right|+\left|\pi_{A}^{L}-\pi_{B}^{L}\right|+\left|\pi_{A}^{U}-\pi_{B}^{U}\right|\right]
\end{aligned}
$$

3) Interval intuitionistic fuzzy entropy [10]

Definition 3 Let $A$ be an interval-valued intuitionistic fuzzy set, then the fuzzy entropy of the interval-intuitionistic fuzzy number $A$ is defined as:

$$
E A=\frac{\min \left\{U_{i q}^{L}, V_{i q}^{L}\right\}+\min \left\{U_{i q}^{U}, V_{i q}^{U}\right\}+\pi_{i q}^{L}+\pi_{i q}^{U}}{\max \left\{U_{i q}^{L}, V_{i q}^{L}\right\}+\max \left\{U_{i q}^{U}, V_{i q}^{U}\right\}+\pi_{i q}^{L}+\pi_{i q}^{U}}
$$


4) Interval intuitionistic fuzzy weighted set settlement

Definition 4 Let $A=\left\{\left\langle x,\left[U_{i}^{L}(x), U_{i}^{U}(x)\right],\left[V_{i}^{L}(x), V_{i}^{U}(x)\right]\right\rangle\right\}$ be an interval-valued intuitionistic fuzzy set, then The interval intuitionistic fuzzy weighted set settlement is:

$$
G_{Z}\left(A_{1}, A_{2}, \cdots, A_{m}\right)=\left(\left[1-\prod_{i=1}^{m}\left(1-U_{i}^{L}\right)^{z}, 1-\prod_{i=1}^{m}\left(1-U_{i}^{U}\right)^{z}\right],\left[\prod_{i=1}^{m}\left(V_{i}^{L}\right)^{z}, \prod_{i=1}^{m}\left(V_{i}^{U}\right)^{z}\right]\right)
$$

\section{Supplier Selection Method Based on Interval Intuitionistic Fuzzy Set for Multi-Attribute Group Decision Making}

\section{1) Description of the problem}

An enterprise need to choose the optimal supplier from alternative suppliers as partners, $A=\left\{A_{1}, A_{2}, \cdots, A_{m}\right\}$ is alternative suppliers, $C=\left\{C_{1}, C_{2}, \cdots, C_{p}\right\}$ is policymakers set, $a=\left\{a_{1}, a_{2}, \cdots, a_{n}\right\}$ is attributes (indicators), weights of attributes $\omega_{j}$ and weights of decision makers $\partial_{k}$ are unknown, decision makers weights vector sets $Z=\left(\partial_{1}, \partial_{2}, \cdots, \partial_{p}\right), \partial_{k} \in[0,1]$ and $\sum_{k=1}^{p} \partial_{k}=1$, the properties of vector sets $\omega=\left\{\omega_{1}, \omega_{2}, \cdots, \omega_{n}\right\}, \omega_{j} \in[0,1]$ and $\sum_{j=1}^{n} \omega_{j}=1$, then the decision makers of all the attributes of each alternative suppliers give evaluation information, and then take a certain method to various alternative sorting or preferred supplier. Attribute value matrix can be expressed as $X_{m \times n}^{k}=\left(x_{i j}^{k}\right)_{m \times n} \cdot x_{i j}^{k}$ is policymakers $C_{k}$ for alternative suppliers $A_{i}$ about attribute $a_{j}$ 's value, by using interval intuitionistic fuzzy number $x_{i j}^{k}=\left(\left[U_{i j}^{L}, U_{i j}^{U}\right],\left[V_{i j}^{L}, V_{i j}^{U}\right]\right)$ said.

\section{2) Determining the weight of decision makers}

The weight of decision makers depends on how reliable the information is. The vaguer the judgment information provided by the decision-maker, the less the decision-maker knows about the decision object, and the less the weight is given. On the contrary, a larger weight is given. In this paper, the entropy and similarity of interval intuitionistic fuzzy number are considered comprehensively from the decision matrix given by decision makers, which can not only guarantee the objectivity of the weight vector, but also reflect the subjective intention of decision makers. Firstly, the interval intuitionistic fuzzy entropy of the decision maker $C_{k}$ on $m$ alternatives under each attribute is determined, and the weight of the decision maker $C_{k}$ is determined $\partial_{k 1}$ :

$$
\begin{gathered}
E\left(A^{k}\right)=\frac{1}{h m} \sum_{q=1}^{h} \sum_{i=1}^{m} e_{i q}^{k} \\
=\frac{1}{h m} \sum_{q=1}^{h} \sum_{i=1}^{m} \frac{\min \left\{U_{i q}^{L}, V_{i q}^{L}\right\}+\min \left\{U_{i q}^{U}, V_{i q}^{U}\right\}+\pi_{i q}^{L}+\pi_{i q}^{U}}{\max \left\{U_{i q}^{L}, V_{i q}^{L}\right\}+\max \left\{U_{i q}^{U}, V_{i q}^{U}\right\}+\pi_{i q}^{L}+\pi_{i q}^{U}} \\
\partial_{k 1}=\frac{1-E\left(A^{k}\right)}{\sum_{k=1}^{p}\left(1-E\left(A^{k}\right)\right)}
\end{gathered}
$$


Then use the interval intuitionistic fuzzy Hamming distance to find the distance between the decision maker $C_{k}$ and other decision matrices, and find the $\partial_{k 2}$ of the decision maker $C_{k}$ :

$$
\begin{gathered}
D\left(A^{k}\right)=\frac{1}{p n m} \sum_{l=1}^{p} \sum_{j=1}^{n} \sum_{i=1}^{m} d\left(\bar{r}_{i j}^{k}, \bar{r}_{i j}^{l}\right) \\
\partial_{k 2}=\frac{1-D\left(A^{k}\right)}{\sum_{k=1}^{p}\left(1-D\left(A^{k}\right)\right)}
\end{gathered}
$$

The weights for policymakers $C_{k}$ eventually: $\partial_{k}=\delta \partial_{k 1}+(1-\delta) \partial_{k 2} . \delta$ can be adjusted according to actual condition, the $\delta \in(0,1)$. This article take the $\delta=0.5$. The weight vector set of the decision maker is obtained as $Z=\left(\partial_{1}, \partial_{2}, \cdots, \partial_{p}\right)$.

\section{3) Determining attribute weight}

As for attribute weight, due to individual differences of decision makers, attribute weight cannot be completely determined, and it is given comprehensively according to the opinions of $\mathrm{p}$ decision makers. This paper determines the attribute weight based on the idea of maximum deviation. The principle of the deviation maximization method is that if the evaluation value of an attribute in each alternative scheme is very small, then the attribute has little effect on the scheme ranking and gives a small weight. On the contrary, the attribute should be given a greater weight. In this paper, the weighted Hamming distance of interval intuitionistic fuzzy number is used to measure the deviation between all attribute values of each alternative scheme.

For the attribute $a_{j}$, the dispersion $d_{j}\left(A_{i}, A_{t}\right)$ between the decision scheme $A_{t}$ and the other decision scheme $A_{t}$ can be defined as:

$$
d_{j}\left(A_{i}\right)=\sum_{t=1}^{m} \omega_{j} d_{j}\left(A_{i}, A_{t}\right)
$$

The sum of the total dispersions for all alternatives is:

$$
S_{j}=\sum_{i=1}^{m} d_{j}\left(A_{i}\right)=\sum_{i=1}^{m} \sum_{t=1}^{m} \omega_{j} d_{j}\left(A_{i}, A_{t}\right)
$$

The attribute weight vector $W=\left(\omega_{1} \cdots \omega_{j}\right)^{\mathrm{T}}$ should be selected so that the total dispersion of each alternative is maximized, so the following optimal model solution is established:

$$
\max D\left(\omega_{j}\right)=\sum_{j=1}^{n} S_{j}=\sum_{j=1}^{n} \sum_{i=1}^{m} \sum_{t=1}^{m} \omega_{j} d_{j}\left(A_{i}, A_{t}\right)
$$

Restrictions:

$$
\begin{gathered}
\sum_{j=1}^{n} \omega_{j}^{2}=1 \\
\omega_{j} \geq 0
\end{gathered}
$$

where $i=1,2, \cdots, m ; j=1,2, \cdots, n ; k=1,2, \cdots, p$. Solving the optimization model (7), you can construct a Lagrangian function:

$$
L_{(\omega, \sigma)}=\sum_{j=1}^{n} \sum_{i=1}^{m} \sum_{t=1}^{m} \omega_{j} d_{j}\left(A_{i}, A_{t}\right)+\sigma\left(\sum_{j=1}^{n} \omega_{j}^{2}-1\right)
$$


Partial derivative $\left\{\begin{array}{l}\frac{\partial L}{\partial \omega}=\sum_{i=1}^{m} \sum_{t=1}^{m} d_{j}\left(A_{i}, A_{t}\right)+2 \sigma \omega_{j}=0 \\ \frac{\partial L}{\partial \sigma}=\sum_{j=1}^{n} \omega_{j}^{2}-1=0\end{array}\right.$; Find a solution: $\omega_{j}=\frac{\sum_{i=1}^{m} \sum_{t=1}^{m} d_{j}\left(A_{i}, A_{t}\right)}{\sqrt{\sum_{j=1}^{n}\left[\sum_{i=1}^{m} \sum_{t=1}^{m} d_{j}\left(A_{i}, A_{t}\right)\right]^{2}}} ;$ Calculate $\omega_{j}$ using Python programming. Traditional weighting vectors also need to satisfy normalized constraints:

$$
\omega_{j}^{*}=\frac{\omega_{j}}{\sum_{j=1}^{n} \omega_{j}}
$$

\section{4) Based on the expansion of VIKOR supplier ranking method}

When selecting suppliers, it is difficult for decision-makers to choose one supplier from many potential suppliers that can fully meet all requirements of the enterprise's evaluation system. Therefore, it is particularly important to choose a good compromise solution in practical decision-making. The VIKOR decision method shows an advantage in this respect. According to the decision matrix, the steps to optimize the decision object according to the VIKOR method are as follows:

a) according to the decision matrix, the positive ideal solution and the negative ideal solution of the intuitionistic fuzzy decision matrix of group interval are defined as respectively: $A^{+}=\left(x_{1}^{+}, x_{2}^{+}, \cdots, x_{j}^{+}\right)$and $A^{-}=\left(x_{1}^{-}, x_{2}^{-}, \cdots, x_{j}^{-}\right)$. Among them, $x_{j}^{+}=\max \left(x_{i j}\right) ; x_{j}^{-}=\min \left(x_{i j}\right) ; i=1,2, \cdots, m ; j=1,2, \cdots, n$; $\max _{1 \leq i \leq m} x_{i j}=\left(\left[\max _{1 \leq i \leq m} U_{i j}^{L}, \max _{1 \leq i \leq m} U_{i j}^{U}\right],\left[\min _{1 \leq i \leq m} V_{i j}^{L}, \min _{1 \leq i \leq m} V_{i j}^{U}\right]\right)$; $\min _{1 \leq i \leq m} x_{i j}=\left(\left[\min _{1 \leq i \leq m} U_{i j}^{L}, \min _{1 \leq i \leq m} U_{i j}^{U}\right],\left[\max _{1 \leq i \leq m} V_{i j}^{L}, \max _{1 \leq i \leq m} V_{i j}^{U}\right]\right)$.

b) Calculate the group utility value $S_{i}$ and individual regret value $R_{i}$ of each alternative scheme:

$$
\begin{array}{r}
S_{i}=\sum_{j=1}^{n} \omega_{j} \frac{\left(x_{j}^{+}-x_{i j}\right)}{\left(x_{j}^{+}-x_{j}^{-}\right)} \\
R_{i}=\max _{j} \omega_{j} \frac{\left(x_{j}^{+}-x_{i j}\right)}{\left(x_{j}^{+}-x_{j}^{-}\right)}
\end{array}
$$

where $\left(x_{j}^{+}-x_{i j}\right)$ and $\left(x_{j}^{+}-x_{j}^{-}\right)$are the differences between two interval intuitionistic fuzzy numbers, and the interval intuitionistic fuzzy Hamming distance is used to calculate the metrics $d\left(x_{j}^{+}, x_{i j}\right)$ and $d\left(x_{j}^{+}, x_{j}^{-}\right)$.

c) Calculate the comprehensive evaluation value of each alternative

$$
Q_{i}=\varepsilon \frac{\left(S_{i}-S^{-}\right)}{\left(S^{+}-S^{-}\right)}+(1-\varepsilon) \frac{\left(R_{i}-R^{-}\right)}{\left(R^{+}-R^{-}\right)}
$$

where $S^{+}=\max _{i}\left\{S_{i}\right\}, S^{-}=\min _{i}\left\{S_{i}\right\}, R^{+}=\max _{i}\left\{R_{i}\right\}, R^{-}=\min _{i}\left\{R_{i}\right\}$.

$\varepsilon$ is the coefficient of decision mechanism, $\varepsilon \in[0,1], \varepsilon>0.5$ means that the supplier is selected according to the decision mechanism of maximizing the util- 
ity of the group; $\varepsilon<0.5$ means that the decision is made according to the decision mechanism of minimizing individual regret; $\varepsilon=0.5$, it means that the strategy is formulated according to the equilibrium situation. In the interval-intuitionistic fuzzy VIKOR method proposed in this paper, the coefficient of decision-making mechanism is chosen to be 0.5 , that is, the optimal supplier is selected according to the mechanism that the decision-makers negotiate to reach consensus.

\section{d) Determine the compromise solution}

According to the order of $S_{i}$ and $R_{i}$ and $Q_{i}$ sorted from small to large, three sorting sequences are obtained. For the candidate suppliers ranked first in $Q_{i}$, the optimal compromise solution is determined according to the following two conditions:

Assume that the first and second schemes are sorted as $A_{1}$ and $A_{2}$, respectively.

Condition 1: $Q_{2}-Q_{1} \geq \frac{1}{m-1}$, where $m$ is the number of scenarios, which represents an acceptable advantage.

Condition 2: If the $A_{1}$ stability of the scheme is accepted by the decision maker during the decision process. $A_{1}$ is also the first scheme in $S_{i}$ and $R_{i}$.

If the above two conditions are met, the optimal solution is $A_{1}$; if the two conditions cannot be met at the same time, a compromise is obtained:

i) If only condition 1 is satisfied, the compromise solution is $A_{1}, A_{2}$;

ii) If only condition 2 is satisfied, based on $Q_{i}-Q_{1} \leq \frac{1}{m-1}$, the maximum $i$ can be obtained, then $A_{1}, A_{2}, \cdots, A_{i}$ are close to the ideal solution.

\section{Case Analysis}

In this paper, an example of an automobile enterprise's choice of parts supplier is taken. The interval intuitionistic fuzzy multi-attribute VIKOR method established in this paper is adopted to help automobile enterprises choose the best auto parts supplier. Weber [11] adopted the method of statistical analysis to collate and analyze 74 literatures published from the 1960s to the 1990s and related to the evaluation and selection of suppliers, and studied and analyzed 23 indicators proposed by Dickson from different perspectives, among which price, quality and service were the most frequently discussed indicators. Car companies now J want to choose the optimal battery supplier to build the cooperation, the existing four alternative battery supplier $A_{i}(i=1,2,3,4)$, J company established three evaluation attributes $a_{j}(j=1,2,3)$ (product price, product quality and service), in order to make decisions scientifically, from procurement, finance, technology, three departments take a human $C_{k}(k=1,2,3)$.

1) supplier selection scheme based on interval intuitionistic fuzzy VIKOR method

Step 1 Determines the initial decision information matrix 
According to their own experience, the three decision makers respectively provide decision information for three attributes of the four suppliers, represented by interval intuitionistic fuzzy number, as shown in Table 1.

Step 2 Determine decision maker weights

The weight set $Z=(0.37,0.35,0.28)$ can be obtained by aggregating the weights of decision makers with $\partial_{k}$ according to Equations (3) and (4).

Step 3 Aggregate group interval intuitionistic fuzzy decision matrix

The interval intuitionistic fuzzy aggregation operator is used to aggregate the decision matrix of three decision makers into a group decision matrix, and the comprehensive evaluation is obtained by value Definition 4 .

$$
\begin{array}{ll}
r_{11}=([0.69,0.78],[0.15,0.20]), & r_{12}=([0.77,0.84],[0.08,0.13]), \\
r_{13}=([0.73,0.78],[0.16,0.19]), & r_{21}=([0.55,0.60],[0.27,0.34]), \\
r_{22}=([0.54,0.63],[0.19,0.25]), & r_{23}=([0.64 .0 .70],[0.18,0.23]), \\
r_{31}=([0.66,0.70],[0.18,0.24]), & r_{32}=([0.77,0.84],[0.11,0.16]), \\
r_{33}=([0.66,0.73],[0.16,0.23]), & r_{41}=([0.61,0.67],[0.21,0.28]), \\
r_{42}=([0.61,0.67],[0.17,0.25]), & r_{43}=([0.70,0.75],[0.08,0.14])
\end{array}
$$

Step 4 Determine attribute weights

According to Formula (6), the total dispersion of all the schemes under each attribute is: $S_{1}=1.07 \omega_{1}, S_{2}=1.69 \omega_{2}, S_{3}=0.90 \omega_{3}$.

The selection of the attribute weight vector $W=\left(\omega_{1} \cdots \omega_{j}\right)^{\mathrm{T}}$ should maximize the total dispersion of each alternative, so the following optimization model is established:

Table 1. Initial decision information matrix.

\begin{tabular}{ccccc}
\hline$C_{k}$ & $A_{1}$ & Product price & product quality & service \\
\hline$C_{1}$ & $A_{1}$ & $([0.70,0.80],[0.15,0.20])$ & $([0.65,0.80],[0.10,0.15])$ & $([0.80,0.85],[0.12,0.15])$ \\
& $A_{2}$ & $([0.65,0.70],[0.25,0.30])$ & $([0.56,0.60],[0.22,0.25])$ & $([0.80,0.83],[0.10,0.14])$ \\
& $A_{3}$ & $([0.80,0.82],[0.10,0.16])$ & $([0.85,0.90],[0.05,0.10])$ & $([0.70,0.75],[0.20,0.25])$ \\
& $A_{4}$ & $([0.75,0.80],[0.15,0.20])$ & $([0.70,0.75],[0.15,0.20])$ & $([0.70,0.70],[0.20,0.25])$ \\
$C_{2}$ & $A_{1}$ & $([0.55,0.65],[0.20,0.25])$ & $([0.78,0.80],[0.10,0.15])$ & $([0.65,0.70],[0.22,0.24])$ \\
& $A_{2}$ & $([0.62,0.65],[0.28,0.35])$ & $([0.69,0.75],[0.15,0.20])$ & $([0.66,0.70],[0.22,0.25])$ \\
& $A_{3}$ & $([0.56,0.60],[0.22,0.25])$ & $([0.80,0.85],[0.12,0.15])$ & $([0.67,0.75],[0.22,0.25])$ \\
& $A_{4}$ & $([0.60,0.65],[0.30,0.35])$ & $([0.73,0.75],[0.15,0.20])$ & $([0.85,0.90],[0.05,0.10])$ \\
$C_{3}$ & $A_{1}$ & $([0.80,0.85],[0.10,0.15])$ & $([0.85,0.90],[0.05 .0 .10])$ & $([0.70,0.75],[0.10,0.20])$ \\
& $A_{2}$ & $([0.20,0.30],[0.30,0.40])$ & $([0.23,0.45],[0.22,0.35])$ & $([0.15,0.35],[0.30,0.40])$ \\
& $A_{3}$ & $([0.50,0.60],[0.30,0.40])$ & $([0.55,0.65],[0.25,0.30])$ & $([0.60,0.65],[0.10,0.20])$ \\
& $A_{4}$ & $([0.30,0.43],[0.20,0.35])$ & $([0.10,0.30],[0.25,0.45])$ & $([0.26,0.35],[0.05,0.10])$ \\
\hline
\end{tabular}




$$
\begin{aligned}
& \max D\left(\omega_{j}\right)=1.07 \omega_{1}+1.69 \omega_{2}+0.90 \omega_{3} \\
& \text { s.t. } \sum_{j=1}^{n} \omega_{j}^{2}=1 \\
& \omega_{j} \geq 0
\end{aligned}
$$

The optimization model is calculated using Python programming, and the attribute weight vector set is obtained: $W=(0.49,0.77,0.41)$. Then normalized according to Formula (10) to obtain $W^{*}=(0.29,0.46,0.25)$.

Step 5 Sorted by the extended VIKOR method to select the optimal solution

According to the first step a) of the VIKOR method, the positive ideal solution and the negative ideal solution are defined:

$x_{j}^{+}=([0.69,0.78],[0.15,0.20]),([0.77,0.84],[0.08,0.13]),([0.73,0.78],[0.08,0.14]) ;$

$x_{j}^{-}=([0.55,0.60],[0.27,0.34]),([0.54,0.63],[0.19,0.25]),([0.64,0.70],[0.18,0.23])$.

Calculate the group utility value $S_{i}$ and the individual regret value $R_{i}$ of each decision scheme by Formula (11), Formula (12) and Formula (13), taking $\varepsilon=0.5$, calculate the comprehensive evaluation value $Q_{i}$ of each alternative scheme $A_{i}$, and obtain $S_{1}=0.17, S_{2}=1, S_{3}=0.39, S_{4}=0.61 ; R_{1}=0.17$, $R_{2}=0.46, R_{3}=0.24, R_{4}=0.35 ; Q_{1}=0, Q_{2}=1.44, Q_{3}=0.37, Q_{4}=0.82$. The sorting results are $S_{1}<S_{3}<S_{4}<S_{2} ; R_{1}<R_{3}<R_{4}<R_{2} ; Q_{1}<Q_{3}<Q_{4}<Q_{2}$. It can be obtained that the value of $Q_{1}$ is at least the scheme $A_{1}$.

\section{2) Sensitivity analysis}

In the interval intuitionistic fuzzy VIKOR method, the decision mechanism $\mathcal{E}$ coefficient has an important influence on the optimal ranking results of the auto parts supplier. This article performs sensitivity analysis by setting different $\varepsilon$ values and observes changes in the choice of compromises to investigate the influence of different $\varepsilon$ values on the evaluation results. Take the value $\varepsilon$ from the interval $[0,1]$ at 0.1 , and conduct 11 experiments in total. The three ranking results obtained by each experiment are the same as those obtained by the above method. That is to say, the best supplier is always $A_{1}$ while setting different decision mechanism $\varepsilon$. This shows the stability of this method.

\section{3) Contrastive analysis with TOPSIS method}

In order to compare the effectiveness and feasibility of the algorithm, I used the interval intuitionistic fuzzy TOPSIS method in reference [12] to make decision on the data of case analysis. And compared with the decision results obtained by the interval intuitionistic fuzzy VIKOR method proposed in this paper. In the interval intuitionistic fuzzy TOPSIS method, using the relative proximity between the alternative suppliers and the ideal solution $K_{i}=d\left(x_{j}^{-}, x_{i j}\right) /\left(d\left(x_{j}^{+}, x_{i j}\right)+d\left(x_{j}^{-}, x_{i j}\right)\right)$ to sort alternate suppliers. And the larger the value $K_{i}$, the closer to the ideal scheme. Where $d\left(x_{j}^{+}, x_{i j}\right)$ and $d\left(x_{j}^{-}, x_{i j}\right)$ are the weighted interval intuitionistic fuzzy Hamming distances of the alternative supplier $A_{i}$ and the positive ideal solution $x_{j}^{+}$and the negative ideal solution $x_{j}^{-}$, respectively. Weight is the weight of each attribute. The equipment evaluation and ranking results obtained by applying interval intui- 
tionistic fuzzy TOPSIS method and VIKOR method are $K_{1}=0.91, K_{2}=0$, $K_{3}=0.73, K_{4}=0.37$ respectively. And The ranking result is $K_{1}<K_{4}<K_{3}<K_{2}$. It can be seen that the ranking results of the alternatives obtained by the interval intuitionistic fuzzy TOPSIS and VIKOR methods are completely consistent, and the optimal scheme is always $A_{1}$, which further validates the effectiveness of the proposed method. In addition, the interval intuitionistic fuzzy VIKOR method can also express the subjective attitude of decision makers, considering the mutual compromise between group benefits and individual regrets, and has greater objectivity and flexibility than the TOPSIS method. And the paper determines the weights of each decision maker based on the interval intuitionistic fuzzy entropy, making the results more objective and in line with the actual situation, while the reference [12] only considers the situation of a single decision maker.

\section{The Summary}

Suppliers are at the input of the supply chain and play a decisive role in the development of the entire supply chain. Aiming at the problem of supplier selection, this paper proposes a decision-making method with interval intuitionistic fuzzy sets for supplier attribute evaluation information. A new VIKOR model of interval intuitionistic fuzzy multi-attribute group decision-making is constructed when attribute weight and decision maker weight are unknown. Then the model is applied to the evaluation and selection of automotive parts suppliers, and the validity of the model is verified by comparing with the relevant methods.

\section{Conflicts of Interest}

The authors declare no conflicts of interest regarding the publication of this paper.

\section{References}

[1] Wan, S., Wang, Q. and Dong, J. (2013) The Extended VIKOR Method for Multi-Attribute Group Decision Making with Triangular Intuitionistic Fuzzy Numbers. Knowledge-Based Systems, No. 52, 65-77. https://doi.org/10.1016/j.knosys.2013.06.019

[2] Pang, J. and Song, P. (2015) Multi-Stage Vendor Selection Based on Fuzzy Group Decision and Extended VIKOR. Statistics and Decision-Making, No. 13, 43-46.

[3] Zhang, L., Wang, J., Zheng, D., Che, F., Shi, C. and Mao, H. (2019) Decision-Making of Equipment Supplier Selection Based on Intuitionistic Fuzzy Entropy and VIKOR. System Engineering and Electronic Technology, No. 3, 1-10.

[4] Zhao, M., Ren, R. and Li, G. (2013) Fuzzy Entropy Group Decision Making Method Based on Interval Intuitionistic Fuzzy Sets. Operations Research and Management, No. 5, 117-121.

[5] Yang, J. and Lai, L. (2013) Supplier Selection Method Considering Decision-Maker's Attitude in Interval Intuitionistic Fuzzy Environment. Logistics Engineering and Management, No. 7, 155-157. 
[6] Yuan, Y., Guan, T., Li, X. and Li, Y. (2014) Supplier Selection Decision Model Based on Hybrid VIKOR Method. Control and Decision, 29, 551-560.

[7] Xu, H., Liu, G. and Zhou, Z. (2017) Interval Intuitionistic Uncertain Linguistic Multi-Attribute Group Decision Model for Risk Identification of Engineering Projects. Journal of Civil Engineering and Management, 34, 68-74.

[8] Atanassovkt, G. (1989) Interval-Valued Intuitionistic Fuzzy Sets. Fuzzy Sets and Systems, 31, 343-349. https://doi.org/10.1016/0165-0114(89)90205-4

[9] Gao, Z. and Wei, C. (2012) For Mulaofinterval-Value Dintu-Itionistic Fuzzy Entropy and Its Applications. Computer Engineering and Application, 48, 53-55.

[10] Wang, P. and Wei, C. (2011) A Construction Method of Interval Intuitionistic Fuzzy Entropy. Computer Engineering and Applications, No. 2, 43-45.

[11] Weber, C.A. and Current, J.R. (1991) Vendor Selection Criteria and Methods. European Journal of Operational Research, 50, 2-18. https://doi.org/10.1016/0377-2217(91)90033-R

[12] Tan, C. (2010) TOPSIS Multi-Attribute Decision Making Based on Interval Value Intuitionistic Fuzzy Sets. Fuzzy Systems and Mathematics, No. 1, 92-97. 